\title{
Addendum to: A comment on some new definitions of fractional derivative
}

\author{
Andrea Giusti $\mathbb{D}$
}

Received: 28 May 2018 / Accepted: 9 June 2018 / Published online: 30 June 2018

(C) Springer Nature B.V. 2018

\begin{abstract}
This is a brief Addendum to Giusti (Nonlinear Dyn 1-7, 2018. https://doi.org/10.1007/ s11071-018-4289-8), whose aim is to properly acknowledge a result that had been obtained in a previous paper, of which the author was unfortunately not aware of until very recently.
\end{abstract}

Keywords Prabhakar function - Mittag-Leffler function - Caputo-Fabrizio derivative - AtanganaBaleanu derivative

Following a recent correspondence with some colleagues, I became aware of the paper [1] by Baleanu and Fernandez.

In [2], I have provided a proof of a general result for Prabhakar integrals [see Theorem 1, Eq. (2.4)] by means of a well- established technique of term-by-term integration of series. From this result, I have then recovered the expansion in Theorem 5 [Eq. (3.6)], as an immediate consequence of the general property in Theorem 1. However, until recently I was not aware that the result that I presented in Theorem 5 had actually been obtained before by Baleanu and Fernandez in [1] [Theorem 2.2, Eq. (16)] by means of the same technique that I employed for the proof of Theorem 1 in [2].

Therefore, considering that Theorem 2.2 in [1] and its derivation, focused solely on the $\mathrm{ABC}$ operator, represent the key point of [1], I firmly believe that this prior work deserves to be properly acknowledged with this Addendum to my original manuscript.

\section{References}

1. Baleanu, D., Fernandez, A.: On some new properties of fractional derivatives with Mittag-Leffler kernel. Commun. Nonlinear Sci. Numer. Simul. 59, 444-462 (2018)

2. Giusti, A.: A comment on some new definitions of fractional derivative. Nonlinear Dyn. 1-7 (2018). https://doi.org/ 10.1007/s11071-018-4289-8. (first online 20 April 2018)

\footnotetext{
A. Giusti $(\varangle)$

Department of Physics and Astronomy, University of Bologna and INFN, Via Irnerio 46, Bologna, Italy e-mail: agiusti@bo.infn.it

A. Giusti

Arnold Sommerfeld Center, Ludwig-Maximilians-Universität, Theresienstraße 37, 80333 Munich, Germany
} 